\title{
Microglial NLRP3 Activity in Alzheimer's Disease
}

\section{Bruno Cabral de Lima Oliveira*}

Instituto de Ensino Superior Presidente Antonio de Almeida Neves-IPTAN, Centro Universitário Presidente Tancredo de Almeida Neves-UNIPTAN, Brazil

* Corresponding author: Bruno Cabral de Lima Oliveira, Instituto de Ensino Superior Presidente Antonio de Almeida NevesIPTAN, Centro Universitário Presidente Tancredo de Almeida Neves-UNIPTAN, Avenida Leite de Castro, 1101, Fábricas, 36301-182, São João del Rei, MG, Brazil, Tel: 55-31-9-9181-6177, E-mail: bcabraloliveira@yahoo.com.br

\begin{abstract}
Alzheimer's Disease (AD) is the major disease leading to dementia. This disease is characterized by the presence of $\beta$-Amyloid $(A \beta)$ extracellular deposits and neurofibrillary tangles, which induce senile plaques formation. Furthermore, inflammation in $A D$ is mainly mediated by innate immunity-related cells, especially microglia. Recent lines of evidence indicate that inflammatory parameters associated with microglia induce mechanisms of pathogenesis in AD. Although the Nucleotide-Binding Domain and Leucine-Rich Repeat Protein 3 (NLRP3) inflammasome has been reported as an important player in this disease, its activity in the Central Nervous System (CNS) during AD is still poorly understood. In this paper the role of microglial cells and the specific activity of NLRP3 in this cell type during $A D$ are highlighted.
\end{abstract}

\section{Keywords}

Alzheimer's disease, NLRP3, Microglia, Neuroinflammation

\section{Introduction}

Alzheimer's Disease (AD) is a disorder leading to dementia, and is one of the greatest public health problems in the $21^{\text {st }}$ century. $A D$ affects more than 25 million individuals worldwide and projections indicate that $A D$ incidence will significantly increase in coming years due to factors such as longer life expectancy and obesity [1]. $A D$ presents multifactorial etiology resulting in progressive cognitive impairment and memory loss [2]. Extracellular deposits of $\beta$-Amyloid (A $\beta$ ) protein and intracellular neurofibrillary tangles of hyperphosphorylated tau proteins are in the core of AD pathogenesis and induce senile plaque formation [2].

Inflammatory responses are another important aspect associated with $A D$ and it is mainly related to my- eloid lineage cells [3]. Unlike other neuroinflammatory diseases, such as Multiple Sclerosis (MS), in which infiltrating T lymphocytes are key players in the Central Nervous System (CNS) inflammation, innate immune response-associated resident cells, including astrocytes and especially microglia, are crucial for the establishment of $A D$ neuroinflammatory parameters [4]. Moreover, inflammasomes are potent innate-associated sensors producing proinflammatory mediators involved in many physiological and pathological events [5-7].

This review outlines the role of microglial cells in the CNS homeostasis as well as in AD pathogenesis. Moreover, this paper reviews aspects associated with inflammatory responses related to NLRP3 inflammasome activity, specifically in microglial cells during AD.

\section{Microglia}

Microglia is hematopoietic derived cell related to innate immunity, which are phenotypically defined as CNS resident macrophages [8]. Among various microglial functions, they play a crucial role in CNS surveillance, monitoring brain environment and eliminating undesired substances, such as cell debris, through phagocytosis, and maintaining CNS integrity [8]. Microglial cells are highly sensitive to changes in the CNS homeostasis [8]. Another important role associated with this cell type is antigen presentation through Major Histocompatibility Complex (MHC) class I and II molecules, activating $\mathrm{T}$ lymphocytes and influencing adaptive immune response [9]. Additionally, microglia have a role in modulating synaptic activities through bidirectional communication with neurons via distinct receptor types, in-

Citation: Oliveira BCL (2017) Microglial NLRP3 Activity in Alzheimer's Disease. Int J Brain Disord Treat 3:019. doi.org/10.23937/2469-5866/1410019

Received: July 29, 2017: Accepted: September 09, 2017: Published: September 11, 2017

Copyright: (c) 2017 Oliveira BCL. This is an open-access article distributed under the terms of the Creative Commons Attribution License, which permits unrestricted use, distribution, and reproduction in any medium, provided the original author and source are credited. 
Table 1: Findings in animal models and clinical studies concerning NLRP3 activity in AD.

\begin{tabular}{|l|l|l|}
\hline Type of study & Reference & Findings \\
\hline Animal model & Heneka, et al. [37] & NLRP3 \\
& Griffin, et al. [28] & IL-1 $\beta$ induces tau protein hyperphosphorylation \\
\hline & Gustin, et al. [42] & A $\beta$ stimuli activate NLRP3 specifically in microglia \\
\hline Wu, et al. [45] & A $\beta$ protofibrils induce NLRP3 activation and IL-1 $\beta$ accumulation in microglia \\
\hline Couturier, et al. [38] & ASC ${ }^{-/}$mice are resistant to experimentally developed AD \\
\hline Clinical study & Ojala, et al. [27] & Increase of IL-18 in AD brain \\
\hline & Heneka, et al. [37] & Increase of Caspase-1 in AD brain \\
\hline & Griffin, et al. [29] & IL-1 $\beta$ is involved in neuronal damage \\
\hline & Saresella, et al. [30] & NLRP3 is upregulated in monocytes of severe AD patients \\
\hline & Dursun, et al. [31] & IL-1 $\beta$ is significantly increased in serum of early-onset AD patients \\
\hline & Chen, et al. [32] & IL-18 is increased in serum of AD patients \\
\hline
\end{tabular}

cluding neurotransmitter, adrenergic and dopaminergic receptors [10]. Resting microglia express low levels of CD45, CD40, CD80, MHC I and II, among others. However, after inflammatory stimulus, these cells significantly increase expression of these molecules, as well as their phagocytic capacity and may stimulate lymphocyte proliferation [11]. Importantly, activated microglia also secrete several cytokines and chemokines, immunomodulating other cell types $[12,13]$.

\section{NLRP3 Inflammasome}

Microglial and other myeloid lineage cells express innate immune response-related receptors [14-16]. Antigen recognition in innate immune response is mediated by pattern recognition receptors, such as NOD-Like Receptor Family Receptors (NLRs), which recognize Pathogen-Associated Molecular Patterns (PAMPs) and Danger-Associated Molecular Patterns (DAMPs) [17]. Inserted in NLR family, inflammasomes are multi-protein complexes formed in the cytosolic compartment of immune cells after stimulation by both PAMPs and DAMPs. The formation of these protein platforms results in caspase- 1 activation and cleavage, as well as subsequent activation of proinflammatory cytokines, such as IL-18 and IL-1ß [18,19].

Among inflammasomes, Nucleotide-binding domain and Leucine-Rich repeat Protein 3 (NLRP3) is the most well-studied component. While other inflammasome types recognize a limited number of DAMPs or PAMPs, a great variety of signals is able to activate NLRP3, highlighting its importance in the immune response against both endogenous and exogenous stimuli, and increasing its clinical relevance [20]. The microbial stimuli that activate NLRP3 include bacterial RNA, hemozoin crystals derived from Plasmodium ssp, viral products, and many others. Endogenous stimuli such as ATP, urate, silica, amyloid protein and cholesterol are some examples of NLRP3 activators [21,22].

Activation of NLRP3 complex is thought to occur in two steps. In the first one, cognate ligands are recognized by innate receptors, such as Toll Like Receptors (TLRs), resulting in translocation of nuclear factor KB (NF$\mathrm{KB})$ to the nucleus and subsequent induction of nlrp3, pro-il-16 and pro-il-18 gene expression. In the second step, different PAMPs and DAMPs may induce NLRP3 machinery oligomerization and activation with recruitment and interaction of Apoptosis-associated Specklike protein containing a CARD (ASC) and procaspase-1, inducing caspase autocleavage and finally cytokine processing [23]. Some pathways have been described as possible mechanisms for the second step. For example, potassium $\left(\mathrm{K}^{+}\right)$efflux is necessary during inflammasome assembly process. Studies have shown that blocking $\mathrm{K}^{+}$ efflux in cell culture inhibits NLRP3 activation after stimulation with several agonists [24]. NLRP3 inflammasome activity is a potent inflammatory mechanism involved in the immune response against various microorganisms, such as Plasmodium spp and Toxoplasma gondii $[25,26]$. However, deregulated inflammasome activity may result in severe pathological processes due to strong inflammatory response.

\section{Microglia, NLRP3 and Alzheimer}

NLRP3 has been linked to pathogenic mechanisms in AD. Table 1 summarizes some important findings in animal models and clinical studies. For example, there is an increased IL-18 level in AD brain patients [27]. Moreover, high levels of IL-1 $\beta$ in the brain induce tau protein hyperphosphorylation in animals [28]. In addition, IL-1 $\beta$ plays an important role in neuronal damage, since this cytokine induces the production of inflammatory factors such as NO (Nitric Oxide) and TNF- $\alpha$ (Tumor Necrosis Factor $\alpha$ ) and these molecules promote the transformation of diffuse amyloid plaques into inflammatory plaques, resulting in cortical neuron damage and cerebral atrophy [29].

Studies have shown that NLRP3 activation may also play a role in the periphery during AD. Monocytes from patients with severe AD have significantly greater NLRP3 activation compared to control groups [30]. Moreover, IL-1 $\beta$ levels are significantly increased in patients with early-onset AD [31]. In another study, Chen and coworkers demonstrated that IL-18, IL-23 and IL-17 are increased in the serum of patients with $A D$ compared to age-matched healthy controls [32].

Polymorphisms in nlrp3 and associated genes have been implicated in AD. Tan and coworkers demonstrate 
that 5'-flanking rs2027432 polymorphism is strongly associated with late-onset AD in Chinese population [33]. In a meta-analysis study, Di Bona and coworkers show that the IL1B +3953 TT genotype is associated with increased AD risk, while IL1B -511 TT genotype only increases AD risk in Caucasians [34]. Furthermore, studies demonstrate that mutations in the IL-18 gene are also associated with the development of AD. For example, individuals carrying the $\mathrm{CC}$ genotype are at increased risk of developing AD [35]. Additionally, -607 C allele and $-137 \mathrm{G}$ allele were implicated in the risk of late-onset $A D$ [36].

Heneka and coworkers recently reported significant increased caspase-1, both in AD human brain and animals that experimentally developed AD [37]. In this study, APP/PS1/NLRP3 ${ }^{-/}$and APP/PS1/caspase- $1 \%$ mice were highly protected from experimental development of $A D$, showing reduced neuroinflammation and decreased amyloid burden [37]. Furthermore, ASC $^{-1-}$ mice with $A D$ model reduced amyloid aggregation, increased astrocyte phagocytosis and reduced memory deficits [38]. Together, these findings strongly suggest a role of NLRP3 inflammasomes in AD pathogenesis.

Regarding specific NLRP3 activity in CNS, recent lines of evidence suggest that inflammasome activities in the nervous tissue are important for the pathogenesis of neurodegenerative diseases, such as MS [39-41]. In $A D$, little is known about NLRP3 activation mechanisms in the CNS during the course of the disease. In experiments using $A \beta$ as NLRP3 stimulus as well as known inflammasome agonists such as ATP and nigericin, Gustin and coworkers indicated that NLRP3 machinery is only present in microglia, at least in some circumstances, and not in astrocytes, reinforcing the crucial role of microglial NLRP3 activity in CNS [42]. However, other studies disagree with these findings and suggest NLRP3 activation in astrocytes after CNS injury. Therefore, further studies are needed to address these issues $[43,44]$.

In primary cultures of microglia, $A \beta$ (1-42) protofibrils induce NLRP3 activation and finally IL-1 $\beta$ accumulation downstream TLR/MyD88 pathway [45]. Additionally, activated microglia surrounding senile plaques in $A D$ brain present high IL-1 $\beta$ production in a Cathepsin B-dependent manner [46].

Autophagy is also an important mechanism for $A \beta$ fibrils degradation and a disability in the autophagy machinery may result in ineffective clearance of misfold proteins, leading to formation of $A \beta$ plaques or neurofibrillary tangles [47]. In microglial cells, $A \beta$ induces autophagy through AMP-Activated Protein Kinase catalytic subunit Alpha 1 (PRKAA1) pathway, inducing autophagy deregulation and NLRP3 inflammasome activation, which suggests that impaired autophagy activity in microglia from AD human brains induces NLRP3-mediated inflammation [48].
Oxidative damage has also been observed in AD patient samples. For example, in postmortem AD brain, there is an increased oxidative damage to DNA compared with age-matched controls [49]. Oxidative damage may modulate NLRP3 inflammasome activation. NLRP3 microglial neurotoxicity mediated by $A \beta$ is associated with Reactive Oxygen Species (ROS) and induces NADPH oxidase-induced ROS production [50]. A $\beta$ peptides induce NADPH oxidase complex activation and stimulate ROS production [51].

\section{NLRP3 Pathway as a Target for AD Treatment}

Because of the key role of NLRP3 pathway in AD, the development of therapeutic strategies targeting its cascade molecules, such as NLRP3, IL-1 1 , IL-18 and Caspase-1 seems highly attractive. Recently, Daniels and coworkers demonstrated that fenamate subclass of Nonsteroidal Anti-Inflammatory Drugs (NSAID) selectively inhibit NLRP3 inflammasome formation in peripheral macrophages. In addition, this treatment prevents memory deficit and neuroinflammation, besides decreasing NLRP3 activation in microglia from treated animals [52]. In another study, the treatment with mefenamic acid, another NSAID drug, reduced neural cell toxicity and protected rats from memory deficits [53]. Furthermore, ibuprofen specifically reduced pro-amyloidogenic $\alpha 1$ antichymotrypsin in vitro and in vivo by suppressing IL-1 $\beta$ [54]. These findings indicate that NSAID targeting NLRP3 pathway may be promising drug candidates for the treatment of patients with AD.

Considering that NLRP3 complex formation can be activated by signals recognized by TLRs, receptor signaling pathways may be promising targets for $A D$ treatment. However, the activation of these receptors may have positive or negative effects during AD. For example, Jin and coworkers reported that TLR4 up regulates proinflammatory cytokines, including IL-1 $\beta$, and is involved in AD animal model progression [55]. On the other hand, another study demonstrated that TLR4, TLR 2 and 9 have an important role in the clearance of $A \beta$ deposits in animal brains [56].

Myd88 is a downstream intracellular adaptor of TLR2, 4 and IL-1R signaling, and activates NF-kb transcription factor to activate proinflammatory cytokine genes. MyD88 deficiency may reduce $A \beta$ load by enhancing the phagocytic capability of microglia [57]. In this line, blocking this molecule could be an interesting target for the inhibition of inflammatory and toxic mechanisms in cells during AD. However, MyD88 seems a controversial target for AD. For example, Michaud and coworkers showed that MyD88-deficient mice present accelerated AD pathology and memory deficits [58]. It is noteworthy that this adapter is central in the signaling of several innate receptors and participates in the activation of many important inflammatory genes against pathogens. Thus, a therapeutic approach targeting 
MyD88 might not be beneficial, inducing susceptibility to infections.

In vitro studies demonstrate that caspase-1 inhibitor Z-YVAD-FMK inhibits the processing of IL-1 $\beta$ via NLRP3 pathway, and attenuates microglial neurotoxicity. This finding suggests that caspase 1 inhibition or IL-1 $\beta$ neutralization can improve the inflammatory process by microglia activation upon $A \beta$ stimuli [50].

The role of IL-1 $\beta$ in AD appears to be more complex than previously thought. Shaftel, et al. demonstrated that IL-1 $\beta$ overexpression lead to robust neuroinflammation in hippocampus of mice, characterized by activation of astrocytes and microfibers as well as secretion of proinflammatory cytokines. Surprisingly, in a mouse model of $A D, 4$ weeks of IL-1 $\beta$ overexpression for 4 weeks protected animals to $A \beta$ pathology [59].

\section{Conclusion}

Neuroinflammatory events have recently been reported as additional parameters to the protein deposition involved in the etiology and pathogenesis of $A D$. Innate immune molecules in microglia are important to outcome $A D$ and may play a crucial role in the establishment of severe inflammation in CNS. Recent studies have indicated the NLRP3 activation pathway as a key immune component for the development of AD. Consequently, NLRP3 could be an important target in therapies for AD as well as other neurodegenerative and neuroinflammatory diseases. To date, no drugs have been developed to directly bind and inhibit NLRP3 activity. Because NLRP3 pathway may influence other important pathways in the immune system, the challenge is to establish a specific strategy to inhibit the activation of this inflammasome and consequently decrease the pathological effects of inflammation in the CNS without altering the functionality of resident cells, such as microglia, or leading the patient to greater vulnerability to infections due to immune deficiency.

\section{Conflict of Interest}

Not existent.

\section{References}

1. Kumar A, Singh A, Ekavali (2015) A review on Alzheimer's disease pathophysiology and its management: An update. Pharmacol Rep 67: 195-203.

2. Selkoe DJ, Hardy J (2016) The amyloid hypothesis of Alzheimer's disease at 25 years. EMBO Mol Med 8: 595-608.

3. Prokop S, Miller KR, Heppner FL (2013) Microglia actions in Alzheimer's disease. Acta Neuropathol 126: 461-477.

4. Heppner FL, Ransohoff RM, Becher B (2015) Immune attack: The role of inflammation in Alzheimer disease. Nat Rev Neurosci 16: 358-372.

5. Thacker SG, Zarzour A, Chen Y, Alcicek MS, Freeman LA et al. (2016) High Density Lipoprotein reduces inflammation from cholesterol crystals by inhibiting inflammasome activation. Immunology 149: 306-319.
6. Meng N, Xia M, Lu YQ, Wang M, Boini KM, et al. (2016) Activation of NLRP3 inflammasomes in mouse hepatic stellate cells during Schistosoma J. Infection. Oncotarget 7: 39316-39331.

7. Kim JH, Sohn HJ, Yoo JK, Kang H, Seong GS, et al. (2016) NLRP-3 inflammasome activation in THP-1 target cells triggered by pathogenic Naegleria fowleri. Infection and Immunity $84: 2422-2428$

8. Nimmerjahn A, Kirchhoff F, Helmchen F (2005) Resting microglial cells are highly dynamic surveillants of brain parenchyma in vivo. Science 308: 1314-1318.

9. Mack CL, Vanderlugt-Castaneda CL, Neville KL, Miller SD (2003) Microglia are activated to become competent antigen presenting and effector cells in the inflammatory environment of the Theiler's virus model of multiple sclerosis. J Neuroimmunol 144: 68-79.

10. Kettenmann H, Hanisch UK, Noda M, Verkhratsky A (2011) Physiology of microglia. Physiol Rev 91: 461-553.

11. Aloisi F (2001) Immune function of microglia. Glia 36: 165179.

12. Hanisch UK (2002) Microglia as a source and target of cytokines. Glia 40: 140-155.

13. Biber K, Vinet J, Boddeke HW (2008) Neuron-microglia signaling: Chemokines as versatile messengers. J Neuroimmunol 198: 69-74.

14. Papageorgiou IE, Lewen A, Galow LV, Cesetti T, Scheffel $\mathrm{J}$, et al. (2016) TLR4-activated microglia require IFN-gamma to induce severe neuronal dysfunction and death in situ. Proc Natl Acad Sci U S A 113: 212-217.

15. Gharagozloo M, Mahvelati TM, Imbeault E, Gris $P$, Zerif E, et al. (2015) The nod-like receptor, NIrp12, plays an anti-inflammatory role in experimental autoimmune encephalomyelitis. J Neuroinflammation 12: 198.

16. Acioglu C, Mirabelli E, Baykal AT, Ni L, Ratnayake A, et al. (2016) Toll like receptor 9 antagonism modulates spinal cord neuronal function and survival: Direct versus astrocyte-mediated mechanisms. Brain Behav Immun 56: 310324.

17. Ting JP, Lovering RC, Alnemri ES, Bertin J, Boss JM, et al. (2008) The NLR gene family: A standard nomenclature. Immunity 28: 285-287.

18. Szabo G, Petrasek J (2015) Inflammasome activation and function in liver disease. Nat Rev Gastroenterol Hepatol 12: 387-400.

19. Chen GY, Nunez G (2010) Sterile inflammation: sensing and reacting to damage. Nat Rev Immunol 10: 826-837.

20. Rathinam VA, Vanaja SK, Fitzgerald KA (2012) Regulation of inflammasome signaling. Nat Immunol 13: 333-342.

21. Tschopp J, Schroder K (2010) NLRP3 inflammasome activation: The convergence of multiple signalling pathways on ROS production? Nat Rev Immunol 10: 210-215.

22. Samstad EO, Niyonzima N, Nymo S, Aune MH, Ryan L, et al. (2014) Cholesterol crystals induce complement-dependent inflammasome activation and cytokine release. $\mathrm{J}$ Immunol 192: 2837-2845.

23. Kim JJ, Jo EK (2013) NLRP3 inflammasome and host protection against bacterial infection. J Korean Med Sci 28: 1415-1423.

24. Jin C, Flavell RA (2010) Molecular mechanism of NLRP3 inflammasome activation. J Clin Immunol 30: 628-631. 
25. Santos ML, Reis EC, Bricher PN, Sousa TN, Brito CF, et al. (2016) Contribution of inflammasome genetics in Plasmodium vivax malaria. Infect Genet Evol 40: 162-166.

26. Gorfu G, Cirelli KM, Melo MB, Mayer-Barber K, Crown D, et al. (2014) Dual role for inflammasome sensors NLRP1 and NLRP3 in murine resistance to Toxoplasma gondii. MBio 5: e01117-e011113.

27. Ojala J, Alafuzoff I, Herukka SK, van Groen T, Tanila H, et al. (2009) Expression of interleukin-18 is increased in the brains of Alzheimer's disease patients. Neurobiol Aging 30: 198-209.

28. Griffin WS, Liu L, Li Y, Mrak RE, Barger SW (2006) Interleukin-1 mediates Alzheimer and Lewy body pathologies. $J$ Neuroinflammation 3: 5.

29. Griffin WS, Stanley LC, Ling C, White L, MacLeod V, et al. (1989) Brain interleukin 1 and S-100 immunoreactivity are elevated in Down syndrome and Alzheimer disease. Proc Natl Acad Sci U S A 86: 7611-7615.

30. Saresella M, La Rosa F, Piancone F, Zoppis M, Marventano I, et al. (2016) The NLRP3 and NLRP1 inflammasomes are activated in Alzheimer's disease. Mol Neurodegener 11: 23.

31. Dursun E, Gezen-Ak D, Hanağası H, Bilgiç B, Lohmann $E$, et al. (2015) The interleukin 1 alpha, interleukin 1 beta, interleukin 6 and alpha-2-macroglobulin serum levels in patients with early or late onset Alzheimer's disease, mild cognitive impairment or Parkinson's disease. J Neuroimmunol 283: 50-57.

32. Chen JM, Jiang GX, Li QW, Zhou ZM, Cheng Q (2014) Increased serum levels of interleukin-18, -23 and -17 in Chinese patients with Alzheimer's disease. Dement Geriatr Cogn Disord 38: 321-329.

33. Tan MS, Yu JT, Jiang T, Zhu XC, Wang HF, et al. (2013) NLRP3 polymorphisms are associated with late-onset Alzheimer's disease in Han Chinese. J Neuroimmunol 265: 91-95.

34. Su F, Bai F, Zhang Z (2016) Inflammatory cytokines and alzheimer's disease: A review from the perspective of genetic polymorphisms. Neurosci Bull 32: 469-480.

35. Bossù $\mathrm{P}$, Ciaramella A, Moro ML, Bellincampi L, Bernardini S, et al. (2007) Interleukin 18 gene polymorphisms predict risk and outcome of Alzheimer's disease. J Neurol Neurosurg Psychiatry 78: 807-811.

36. Yu JT, Tan L, Song JH, Sun YP, Chen W, et al. (2009) Interleukin-18 promoter polymorphisms and risk of late onset Alzheimer's disease. Brain Res 1253: 169-175.

37. Heneka MT, Kummer MP, Stutz A, Delekate A, Schwartz $S$, et al. (2013) NLRP3 is activated in Alzheimer's disease and contributes to pathology in APP/PS1 mice. Nature 493 : 674-678.

38. Couturier J, Stancu IC, Schakman O, Pierrot N, Huaux F, et al. (2016) Activation of phagocytic activity in astrocytes by reduced expression of the inflammasome component ASC and its implication in a mouse model of Alzheimer disease. J Neuroinflammation 13: 20.

39. Jha S, Srivastava SY, Brickey WJ, locca H, Toews A, et al. (2010) The inflammasome sensor, NLRP3, regulates CNS inflammation and demyelination via caspase-1 and interleukin-18. J Neurosci 30: 15811-15820.

40. Shao BZ, Wei W, Ke P, Xu ZQ, Zhou JX, et al. (2014) Activating cannabinoid receptor 2 alleviates pathogenesis of experimental autoimmune encephalomyelitis via activation of autophagy and inhibiting NLRP3 inflammasome. CNS Neurosci Ther 20: 1021-1028.

41. Burm SM, Peferoen LA, Zuiderwijk-Sick EA, Haanstra KG, Hart BA, et al. (2016) Expression of IL-1beta in rhesus EAE and MS lesions is mainly induced in the CNS itself. J Neuroinflammation 13: 138.

42. Gustin A, Kirchmeyer M, Koncina E, Felten P, Losciuto S, et al. (2015) NLRP3 Inflammasome is expressed and functional in mouse brain microglia but not in astrocytes. PLoS One 10: e0130624.

43. Alfonso-Loeches S, Urena-Peralta JR, Morillo-Bargues MJ, Oliver-De La Cruz J, Guerri C, et al. (2014) Role of mitochondria ROS generation in ethanol-induced NLRP3 inflammasome activation and cell death in astroglial cells. Front Cell Neurosci 8: 216.

44. Johann S, Heitzer M, Kanagaratnam M, Goswami A, Rizo $\mathrm{T}$, et al. (2015) NLRP3 inflammasome is expressed by astrocytes in the SOD1 mouse model of ALS and in human sporadic ALS patients. Glia 63: 2260-2273.

45. Wu Z, Sun L, Hashioka S, Yu S, Schwab C, et al. (2013) Differential pathways for interleukin-1beta production activated by chromogranin A and amyloid beta in microglia. Neurobiol Aging 34: 2715-2725.

46. Correia SC, Resende R, Moreira PI, Pereira CM (2015) Alzheimer's disease-related misfolded proteins and dysfunctional organelles on autophagy menu. DNA Cell Biol 34: 261-273.

47. Wang $Y$, Martinez-Vicente M, Kruger U, Kaushik S, Wong E, et al. (2009) Tau fragmentation, aggregation and clearance: The dual role of lysosomal processing. Hum Mol Genet 18: 4153-4170.

48. Cho MH, Cho K, Kang HJ, Jeon EY, Kim HS, et al. (2014) Autophagy in microglia degrades extracellular beta-amyloid fibrils and regulates the NLRP3 inflammasome. Autophagy 10: 1761-1775.

49. Mecocci P, MacGarvey U, Beal MF (1994) Oxidative damage to mitochondrial DNA is increased in Alzheimer's disease. Ann Neurol 36: 747-751.

50. Parajuli B, Sonobe $\mathrm{Y}$, Horiuchi $\mathrm{H}$, Takeuchi $\mathrm{H}$, Mizuno $\mathrm{T}$, et al. (2013) Oligomeric amyloid beta induces IL-1beta processing via production of ROS: Implication in Alzheimer's disease. Cell Death Dis 4: e975.

51. Bianca VD, Dusi S, Bianchini E, Dal Prà I, Rossi F (1999) beta-amyloid activates the $\mathrm{O}-2$ forming NADPH oxidase in microglia, monocytes, and neutrophils. A possible inflammatory mechanism of neuronal damage in Alzheimer's disease. J Biol Chem 274: 15493-15499.

52. Daniels MJ, Rivers-Auty J, Schilling T, Spencer NG, Watremez W, et al. (2016) Fenamate NSAIDs inhibit the NLRP3 inflammasome and protect against Alzheimer's disease in rodent models. Nat Commun 7: 12504.

53. Joo Y, Kim HS, Woo RS, Park CH, Shin KY, et al. (2006) Mefenamic acid shows neuroprotective effects and improves cognitive impairment in in vitro and in vivo Alzheimer's disease models. Mol Pharmacol 69: 76-84.

54. Morihara T, Teter B, Yang F, Lim GP, Boudinot S, et al. (2005) Ibuprofen suppresses interleukin-1beta induction of pro-amyloidogenic alpha1-antichymotrypsin to ameliorate beta-amyloid (Abeta) pathology in Alzheimer's models. Neuropsychopharmacology 30: 1111-1120.

55. Jin JJ, Kim HD, Maxwell JA, Li L, Fukuchi K (2008) Toll-like receptor 4-dependent upregulation of cytokines in a transgenic mouse model of Alzheimer's disease. J Neuroinflam- 
mation 5: 23.

56. Tahara K, Kim HD, Jin JJ, Maxwell JA, Li L, et al. (2006) Role of toll-like receptor signalling in Abeta uptake and clearance. Brain 129: 3006-3019.

57. Lim JE, Kou J, Song M, Pattanayak A, Jin J, et al. (2011) MyD88 deficiency ameliorates $\beta$-amyloidosis in an animal model of Alzheimer's disease. Am J Pathol 179: 1095-1103.
58. Michaud JP, Richard KL, Rivest S (2011) MyD88-adaptor protein acts as a preventive mechanism for memory deficits in a mouse model of Alzheimer's disease. Mol Neurodegener 6: 5 .

59. Shaftel SS, Kyrkanides S, Olschowka JA, Miller JN, Johnson RE, et al. (2007) Sustained hippocampal IL-1 beta overexpression mediates chronic neuroinflammation and ameliorates Alzheimer plaque pathology. J Clin Invest 117: 1595-1604. 